\title{
Endoscopic Assistance in Retrosigmoid Transmeatal Approach for the Management of Postoperative Cerebrospinal Fluid Fistula
}

\author{
Jasmit Singh $^{1}$ Rocio Evangelista Zamora ${ }^{1}$ Marcos Tatagiba ${ }^{1}$
}

${ }^{1}$ Department of Neurosurgery, Eberhard-Karls-University of Tübingen, Tübingen, Germany

Indian J Neurosurg 2018;7:168-170

Over the past several decades, the outcome of treatment for patients with vestibular schwannoma (VS) has significantly improved. The goal of the treatment in the early 20th century was to resect the tumor without perioperative mortality. However, with the improvement of diagnostic tools (early-stage diagnosis), advances in anesthetic care, introduction of microsurgical techniques, and intraoperative monitoring, mortality and neurologic morbidity have been significantly reduced without compromising the extent of tumor resection. Therefore, it became possible to preserve normal function of cranial nerves, including the facial nerve and sometimes achieve hearing preservation. Retromastoid approach is currently the most commonly used surgical corridor to VSs. This technique has some limitations in the case of intracanalicular tumors. ${ }^{1}$ This is overcome by drilling the internal acoustic meatus. Transmeatal approach is an essential element of retrosigmoid surgery whenever a VS has some intracanalicular components. ${ }^{1}$ To expose this component and intracanalicular portions of cranial nerves VII and VIII, posterior wall of the internal auditory canal (IAC) needs to be drilled. Because of the optics of the operating microscope, there are hidden corners that are beyond the vision of the operating surgeon. ${ }^{2}$ Opened air cells of the temporal bone due
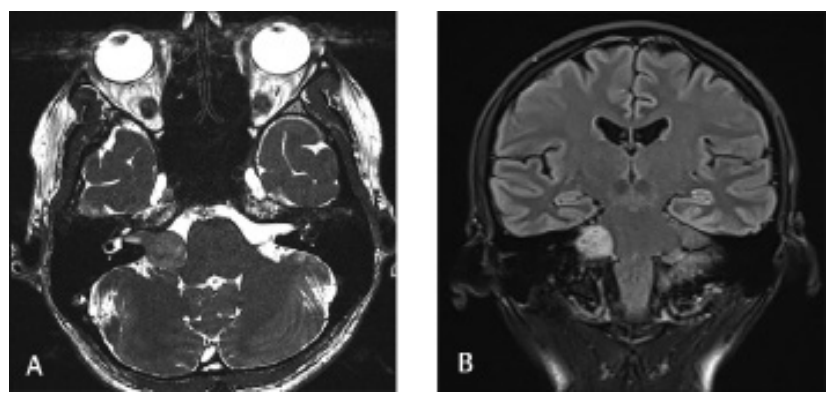

Fig 1 Preoperative MRI brain axial (A) and coronal view (B) showing contrast enhancing lesion in right CPA, extending into the IAC, representing VS.
Address for correspondence Jasmit Singh, MCh, Department of Neurosurgery, Eberhard-Karls-University of Tübingen, Tübingen, Germany (e-mail: jasmeetthukral80@gmail.com).

to removal of the posterior wall of the IAC, which are difficult to visualize, may lead to postoperative leakage of cerebrospinal fluid (CSF). The aim of this technical note is to highlight the importance of using endoscope in management of postoperative CSF leak. Endoscopic guidance provides the operator with a detailed insight in the anatomy of the IAC down to its fundus. ${ }^{2}$ It is helpful in identification of exposed cells that are missed with operating microscope, and it also improves closure of the cells, thus decreasing the risk of CSF fistula. ${ }^{3}$

A 35-year-old woman presented to our outpatient clinic with history of right-side hearing loss and tinnitus. The hearing loss was progressive, and the tinnitus became increasingly annoying off late. The patient sought medical consultation mainly for hearing loss as she could barely hear from right side. Physical examination revealed sensorineural hearing loss on right side along with subtle cerebellar signs on right side. Magnetic resonance imaging (MRI) studies with and without contrast showed a right-side contrast-enhancing tumor extending into the internal auditory canal, compatible with a VS T4a (-Figs. 1 and $\mathbf{2}$ ). The retrosigmoid transmeatal

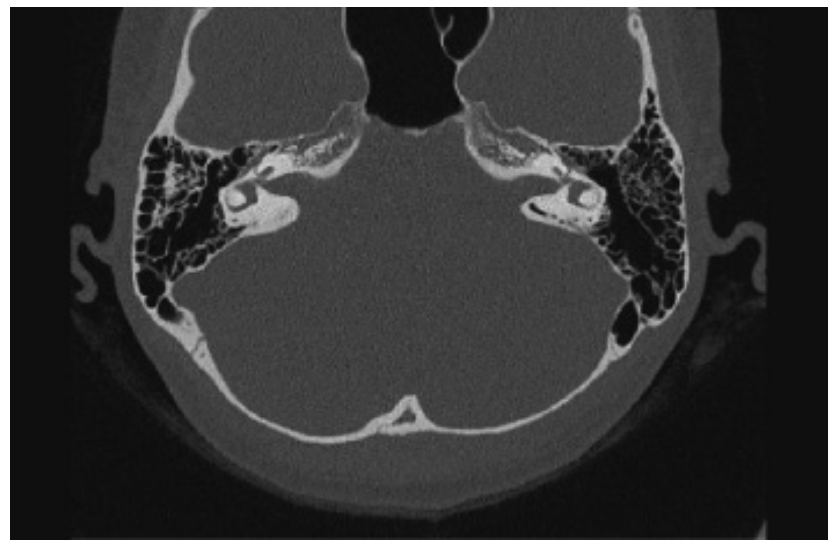

Fig 2 Preoperative CT scan of temporal bone axial view showing the area of IAC. received

September 27, 2017

accepted after revision

March 13, 2018

published online

August 1, 2018
DOI https://doi.org/

10.1055/s-0038-1649559.

ISSN 2277-954X.
¿C2018 Neurological Surgeons'

Society of India
License terms

(요 (1) $\Theta \circledast$ 
approach was performed in semisitting position, under electrophysiological monitoring. A standard 3-cm retromastoid craniotomy was done exposing the transverse-sigmoid sinus junction. The exposed mastoid cells were generously waxed. After opening the dura and cistern magna, the tumor and the Tubingen line were identified. Drilling of the posterior wall of IAC was performed to locate the nerves laterally. This was followed by gradual arachnoid dissection of the tumor along with internal decompression using Cavitron ultrasonic aspirator (CUSA, Integra Life Sciences). The tumor, including the intrameatal part, was resected without any significant change in the electrophysiologic recordings. As a protocol, a significant time was invested in sealing off the opened air cells with bone wax; it was reinforced with muscle piece and fibrin glue, and water tight dural closure was obtained. Postoperative course was uneventful, and the patient remained neurologically intact, with no evidence of facial paresis. However, she complained of watery discharge from nostril on fifth postoperative day. A computed tomographic (CT) of the brain was done to rule out hydrocephalus and biochemical analysis of fluid confirmed CSF ( $\bullet$ Fig. 3 ). She was initially managed with lumbar drain. She improved temporarily and the leak subsided, only to reappear after removal of drain. Eventually a reexploration was planned. The dura was found intact with no evidence of CSF leak, and the mastoid cells appeared well sealed. The dura was opened and cerebellum gently retracted. Under high microscopic magnification, the IAC appeared well sealed with the muscle piece; it was removed, but there was no clear evident site of leak. A 4-mm 45-degree endoscope was introduced to inspect the region of the internal auditory canal and the drilled area to identify the culprit site of CSF leak. There was evidence of two small air cells on the posterior aspect of the drilled IAC area, which were not seen with microscope due to its inherent optics ( - Fig. 4). Using a free-hand technique with scope in one hand, the air cells were waxed under direct visualization. After satisfactory closure, a piece of muscle was used to seal the area, reinforced with fibrin glue, followed by a standard closure. The patient recovered well as was relieved of her CSF leak

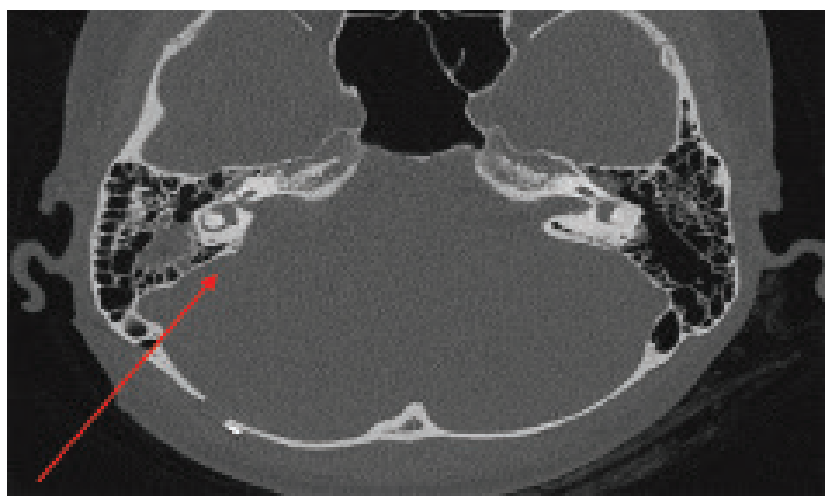

Fig 3 Postoperative CT scan of temporal bone showing drilled area of IAC with evidence of opened air cells (arrow).

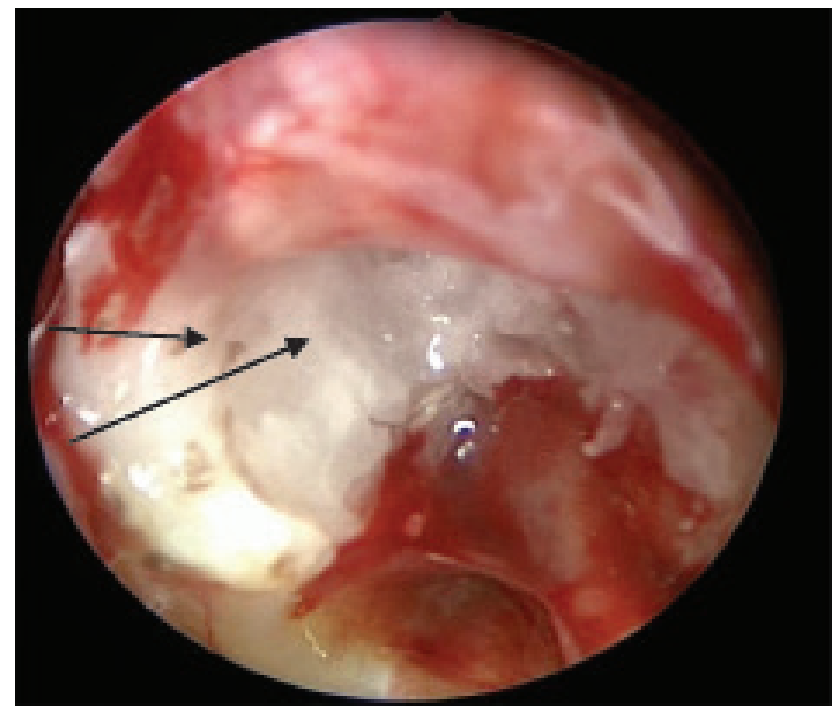

Fig. 4 Intraoperative photograph (endoscopic view) showing two small open air cells (arrows) on the posterior wall of drilled IAC. Note that the bone wax does not properly cover both openings (corresponding with the postoperative $\mathrm{CT}$ image of temporal bone.

Postoperative CSF leak rates reported in the literature range from 2 to $30 \%$; the average leak rate appears to be approximately $10 \%{ }^{4}$ The numerous techniques described in the literature to prevent CSF leak in VS surgery are a testament to the difficulty of the problem and to the lack of universal success with any single method. The opened air cells/petrous bone serve as potential sites for egress of CSF out along their natural pathways. ${ }^{5}$ Presence of late-onset CSF leak in form of rhinorrhea is less likely to respond to medical management., ${ }^{5,6}$ On exploration the mastoid air cells and the dura are carefully examined for any evidence of a breach. Once the dura is opened, the focus is concentrated at the IAC and the part of drilled petrous bone. Even with the use of high-end latest microscopes, it is not routinely possible to look into the blind corners of the auditory canal. There is always a possibility of unobliterated air cells that could not be visualized during first surgery. This is where a side viewing rigid neuroendoscope complements a microscope. Endoscopes increase the light intensity while approaching an object, as such permit clear depiction of details in close-up positions and, due to the angled optics, extend the viewing angle without the need for retraction. Endoscopes thus represent an ideal adjunct especially in retromastoid transmeatal approach in which the IAC is drilled. ${ }^{2}$

This patient had perfect functional result after tumor resection, but unfortunately she developed CSF leak. The identification and sealing of site of CSF leak was possible with use of side viewing rigid endoscope. Modern neuroendoscopy provides a wide angle of view and greater depth of focus with high magnification and illumination. The authors recommend use of endoscope not only for enhancing the resection rate but also for better closure of the air cells under direct visual control. 


\section{Conflict of Interest}

None.

\section{References}

1 Turek G, Cotúa C, Zamora RE, Tatagiba M; GrzegorzTurek et al. Endoscopic assistance in retrosigmoid transmeatal approach to intracanalicular vestibular schwannomas-an alternative for middle fossa approach. Technical note. Neurol Neurochir Pol 2017;51(2):111-115

2 Tatagiba MS, Roser F, Hirt B, Ebner FH. The retrosigmoid endoscopic approach for cerebellopontine-angle tumors and microvascular decompression. World Neurosurg 2014;82(6, Suppl):S171-S176
3 Tatagiba M, Matthies C, Samii M. Microendoscopy of the internal auditory canal in vestibular schwannoma surgery. Neurosurgery 1996;38(4):737-740

4 Mangus BD, Rivas, A, Yoo, MJ , et al. Management of CSF leaks after vestibular schwannoma surgery.

5 Dorward NL, Palazzo FF, Illingworth RD, Cheeseman AD. A technique to reduce CSF leakage after acoustic neuroma surgery. Br J Neurosurg 1997; 11(5):418-420

6 Azad T, Mendelson ZS, Wong A, Jyung RW, Liu JK. Fat graft-assisted internal auditory canal closure after retrosigmoid transmeatal resection of acoustic neuroma: technique for prevention of cerebrospinal fluid leakage. J Clin Neurosci 2016;24:124-127 